\title{
Câncer de próstata e testosterona: riscos e controvérsias
}

\author{
Prostate carcinoma and testosterone: risks and controversies
}

Ernani Luis Rhoden', Márcio Augusto Averbeck ${ }^{2}$

Disciplina de Urologia, Departamento de Cirurgia, Universidade Federal de Ciências da Saúde de Porto Alegre (UFCSPA), Porto Alegre, RS, Brasil 1 Universidade Federal de Ciências da Saúde de Porto Alegre (UFCSPA) Porto Alegre, RS, e Conselho Nacional de Desenvolvimento Científico e Tecnológico (CNPq), Brasília, DF, Brasil 2UFCSPA, Porto Alegre, RS, Brasil
Correspondência para: Ernani Luis Rhoden

Rua Dr. Florencio Ygartua, 288, sala 504

90430-010 - Porto Alegre, RS,

Brasil

ernanirh@terra.com.br

Recebido em 19/Agos/2009 Aceito em 31/Out/2009

\section{RESUMO}

O hipogonadismo é uma síndrome clínica e bioquímica que pode estar associada a um prejuízo significativo na qualidade de vida $(\mathrm{QoL})$ do homem. Com o aumento na expectativa de vida e sobrevida do câncer prostático ( $\mathrm{CaP}$ ), espera-se um número maior de diagnósticos de hipogonadismo em homens submetidos ao tratamento potencialmente curativo do CaP. Apesar da contraindicação clássica do emprego de terapia de reposição com testosterona (TRT) em homens com diagnóstico ou suspeita de $\mathrm{CaP}$, não há evidência convincente de que a normalização dos níveis de testosterona séricos em homens com baixos níveis seja deletéria. Em poucas séries de casos que descreveram aTRT após o tratamento do $\mathrm{CaP}$, não houve casos de progressão clínica ou bioquímica do tumor. Não obstante a literatura seja limitada, os dados disponíveis sugerem que aTRT pode ser considerada em homens hipogonádicos selecionados, previamente tratados curativamente para o $\mathrm{CaP}$ de baixo risco e sem evidência de doença ativa. Arq Bras Endocrinol Metab. 2009;53(8):956-62

\section{Descritores}

Neoplasia prostática; terapia de reposição de testosterona; prostatectomia radical; braquiterapia e câncer de próstata; tratamento de câncer de próstata

\begin{abstract}
Hypogonadism is a clinical and biochemical syndrome which may cause significant detriment in the quality of life. With the increase in life expectancy and prostate cancer survival a significant increase in the number of men with hypogonadism who have undergone presumably curative treatment for $\mathrm{PCa}$ is anticipated. Despite the widespread contraindication of testosterone in men with known or suspected prostate cancer, there is no convincing evidence that the normalization of testosterone serum levels in men with low, but not castrate levels, is deleterious. Although further studies are necessary before definitive conclusions can be drawn, the available evidence suggests that testosterone replacement therapy can be cautiously considered in selected hypogonadal men treated with curative intent for low risk prostate cancer and without evidence of active disease. Arq Bras Endocrinol Metab. 2009;53(8):956-62

Keywords

Prostatic neoplasms; testosterone replacement therapy; radical prostatectomy; brachytherapy and prostate cancer; prostate cancer treatment
\end{abstract}

\section{INTRODUÇÃO}

$\mathrm{O}$ hipogonadismo é uma síndrome clínica e bioquímica caracterizada por sinais e sintomas típicos e níveis baixos de testosterona sérica que podem causar prejuízo importante na qualidade de vida (QoL) e afetar negativamente a função de múltiplos órgãos e sistemas (1). A entidade conhecida como hipogonadismo idiopático do adulto é caracterizada por níveis séricos baixos de testosterona, diminuição da libido, depressão, dimi- nuição da massa muscular e densidade óssea, anemia e fadiga. A suplementação com testosterona pode beneficiar os pacientes mediante melhora na função sexual, aumento da densidade óssea e massa "magra”, estimulação da eritropoiese e melhora na disposição física (2).

Evidências preliminares associaram a síndrome de deficiência de testosterona (TDS) com mortalidade prematura e com um número de comorbidades, incluindo diabetes e síndrome metabólica. Essas associações po- 
dem causar implicações econômicas significativas e prejuízo importante na QoL (1,3-5).

Apesar de a relação entre andrógenos e o desenvolvimento de carcinoma prostático estar ainda em estudo, os dados disponíveis demonstram risco semelhante de desenvolvimento de câncer prostático $(\mathrm{CaP})$ em homens hipogonádicos tratados ou não com suplementação de testosterona. De outro lado, em um paciente com história de $\mathrm{CaP}$ tratado com objetivo curativo e diagnóstico de hipogonadismo, a preocupação permanece sobre o risco de a TRT promover recorrência de focos tumorais remanescentes. Essa preocupação é lógica e teoricamente válida, mas ainda não foi adequadamente estudada (2).

Desde que Huggins descreveu que a castração causava regressão das metástases do $\mathrm{CaP}$, muito debate sobre a associação entre testosterona e $\mathrm{CaP}$ tem ocorrido $(1,6)$. Primeiramente, surgiu a hipótese de que os homens com níveis endógenos mais elevados de testosterona poderiam ter um risco aumentado para o desenvolvimento do CaP. Mesmo que a maioria dos estudos (7-12) não tenha encontrado correlação entre testosterona sérica e o futuro desenvolvimento de $\mathrm{CaP}$, dois estudos $(13,14)$ demonstraram uma correlação positiva entre os níveis de andrógenos e o CaP, sugerindo uma potencial implicação da TRT com o risco de desenvolvimento do CaP. Posteriormente, verificou-se que a taxa de $\mathrm{CaP}$ em estudos de homens submetidos à terapia de reposição com testosterona (TRT) foi semelhante àquela da população geral (15).

O objetivo deste artigo é revisar, de forma crítica, a literatura existente sobre TRT e CaP. As quatro séries de casos disponíveis sobre pacientes que receberam TRT após o tratamento potencialmente curativo do $\mathrm{CaP}$ serão expostas e discutidas.

\section{TRT APÓS 0 TRATAMENTO POTENCIALMENTE CURATIVO DO CÂNCER DE PRÓSTATA}

A disponibilidade do antígeno prostático específico (PSA), juntamente com o exame digital da próstata, como forma de rastreamento para o $\mathrm{CaP}$, facilitou o diagnóstico de tumores localizados ao órgão em um número significativo de homens. Em função do diagnóstico precoce por meio da indicação de biópsia prostática transretal guiada por ecografia, espera-se diagnosticar mais casos de hipogonadismo em homens previamente submetidos ao tratamento curativo do $\mathrm{CaP}$ (16). $\mathrm{O}$ manejo desses pacientes ainda é controverso. O Food and Drug Administration (FDA) declarou que a TRT é contraindicada a homens com CaP conhecido ou suspeito, mas não embasa essa contraindicação. No "Clinical Practice Guideline" a Sociedade Americana de Endocrinologia se posiciona contra a TRT em homens com $\mathrm{CaP}$, mas reconhece que as evidências disponíveis contra a TRT neste contexto são de qualidade muito baixa (17).

Modelos animais mostraram que o aumento nos níveis de testosterona plasmática se correlaciona positivamente com o crescimento de carcinoma prostático em tecido de origem humana (18). Entretanto, estudos clínicos sobre a administração de testosterona em homens com $\mathrm{CaP}$ mostraram resultados controversos. Um grande número de hipóteses surgiu para explicar essa situação (1). Huggins descreveu (1941) que a administração diária de testosterona a dois pacientes (um dos quais já havia sido castrado) com CaP metastático levou a aumento na fosfatase ácida (6). Em um relato de caso, Pearson (19) descreveu um paciente com dor óssea por metástases que se tornou assintomático após a administração diária de testosterona, um efeito que durou por 9 meses. Fowler e Whitmore (20) também executaram um estudo interessante em 52 pacientes com metástases ósseas de CaP que receberam TRT. Quarenta e cinco pacientes apresentaram progressão tumoral.

Prout e Brewer (21) administraram testosterona diariamente por uma mediana de 13 dias a 26 homens com CaP localmente avançado ou metastático. Seis pacientes haviam sido submetidos à castração ou a outro tratamento de deprivação hormonal anteriormente. Os autores descreveram que as variações nos níveis de fosfatase ácida foram "extremamente variáveis" e que a maioria dos pacientes "apresentou uma melhora na percepção de bem-estar e alguns relataram uma vaga diminuição da dor".

Esses dados controversos podem ser interpretados com base na hipótese de que a testosterona estimula o tecido prostático de maneira dose-dependente apenas até um ponto de "saturação" $(1,20,22)$. Tal teoria é fundamentada pelas observações de que a administração de testosterona causa progressão tumoral em homens previamente castrados, tem um mínimo efeito no PSA e volume prostático em homens hipogonádicos $(23,24)$ e não afeta esses parâmetros em homens normais $(25,26)$.

De maneira complementar, Marks e cols. (23) demonstraram que, em um ensaio clínico randomizado controlado de TRT ou placebo por seis meses em ho- 
mens hipogonádicos, a TRT aumenta significativamente os níveis séricos de testosterona, tem um mínimo efeito no PSA e não tem efeito nos níveis de testosterona no tecido prostático, diidrotestosterona, histologia, expressão gênica, incidência de câncer ou severidade. Esses dados sugerem que há um uptake mínimo pela próstata da testosterona exógena administrada aos homens hipogonádicos. Isso embasa a hipótese de que, após alcançar níveis séricos mínimos necessários para a manutenção da expressão gênica, a suplementação adicional de testosterona não iria levar a efeitos androgênicos adicionais mediados por receptor. Contudo, deve ser ressaltado que a maioria desses pacientes apresentava níveis de testosterona sérica limítrofes e que eles foram tratados por somente seis meses.

Há poucos casos relatados na literatura que demonstram uma suposta ativação de CaP latente (subclínico) em homens hipogonádicos que receberam TRT (27). Em um estudo, Curran e Bihrle (28) descreveram o caso de um paciente hipogonádico que recebeu TRT e, após seis meses, teve nódulo prostático palpável e aumento de vintes vezes no nível de PSA (alcançando $55,2 \mathrm{ng} / \mathrm{mL}$ ). Essa associação é fraca para tecer considerações sobre causalidade.

Mais recentemente, foi sugerido que a progressão clínica aguda observada em algumas séries após a administração de agonistas do LHRH (luteinizing hormone-releasing hormone) poderia ter sido ocasionada pelo aumento nos níveis de testosterona que esses agentes causam na primeira semana de tratamento (efeito flare) (29). Contudo, as discrepâncias na frequência e na severidade dos eventos clínicos e o fato de que os estudos falharam em detectar um aumento no PSA durante o flare nos levam a questionar tal hipótese $(30,31)$.

\section{TRT EM HOMENS SUBMETIDOS À PROSTATECTOMIA RADICAL PARA 0 TRATAMENTO DO CARCINOMA PROSTÁTICO LOCALIZADO}

Em relação ao uso de TRT após prostatectomia radical (PR), Kaufman e Graydon (16) relataram uma série de casos com 7 pacientes que receberam reposição de testosterona no pós-operatório, incluindo 4 com níveis de testosterona inferiores a $100 \mathrm{ng} / \mathrm{mL}$. Em seis casos, as margens cirúrgicas foram negativas e, em um caso, a margem apical foi comprometida (mas o PSA pósoperatório foi indetectável). O escore de Gleason foi de 6 ou 7. Cinco homens foram tratados com reposi- ção transdérmica e dois, com injeções intramusculares. Todos os pacientes receberam aconselhamento sobre o risco potencial da administração de testosterona exógena, mesmo com a aparente cura da neoplasia. Os níveis de PSA foram avaliados periodicamente após o início da TRT. Nesta série de casos, a TRT foi benéfica e segura, sem evidência de recorrência local ou metástases a distância, com um acompanhamento que variou de 1 a $12 \operatorname{anos}(16)$.

Outro estudo sobre TRT após PR foi conduzido por Agarwal e Oefelein (2). Os autores realizaram uma revisão retrospectiva de pacientes com carcinoma prostático localizado que foram subsequentemente tratados com TRT, em função de hipogonadismo. Dez homens hipogonádicos sintomáticos, que foram previamente submetidos à prostatectomia radical retropúbica, foram incluídos. Não havia evidência de doença neoplásica ativa por meio de critérios clínicos e PSA. Todos os pacientes receberam consentimento informado e informações sobre o potencial risco da TRT após PR. Os pacientes receberam testosterona exógena por aplicações tópicas, transdérmicas ou intramusculares e foram seguidos em intervalos regulares (a cada dois meses) com determinação do PSA sérico e níveis de testosterona. A idade média dos pacientes foi 64,3 anos. A média do PSA pré-operatório foi de $7,0 \mathrm{ng} / \mathrm{mL}(5,8$ a 12,6$)$. $\mathrm{O}$ escore de Gleason obtido do exame anatomopatológico definitivo variou de 6 a 8 , com Gleason $3+3$ em dois pacientes, Gleason $3+4$ em cinco pacientes, Gleason $4+3$ em dois pacientes e Gleason $4+4 \mathrm{em} \mathrm{um}$ paciente. O PSA pós-operatório foi inferior a $0,1 \mathrm{ng} / \mathrm{mL}$ e a média da testosterona sérica pré-TRT foi de 197 ng/dL. A mediana da duração da TRT foi de 19 meses. Durante o curso da TRT, nenhum paciente teve recorrência do PSA. A testosterona total aumentou significativamente após o início da TRT, de uma média de 197 (95\% IC 145 a 248$)$ a $591 \mathrm{ng} / \mathrm{dL}(95 \%$ IC 469 a 713$)$ ( $\mathrm{p}=0,0002)$. Os autores concluíram que, em acompanhamento rigoroso, os homens hipogonádicos curados do CaP podem ser tratados com TRT de forma segura com resultados benéficos. Contudo, reconheceu-se que um ensaio clínico randomizado, placebo-controlado, para avaliar a TRT em homens hipogonádicos pós-PR é necessário.

Mais recentemente, Khera e cols. (32) relataram um estudo retrospectivo sobre TRT após prostatectomia radical (PR). Apenas pacientes com valores indetectáveis de PSA e margens cirúrgicas negativas no exame anatomopatológico definitivo foram candidatos à TRT. 
Cinquenta e sete homens, com idades entre 53 e 83 anos (média $=64$ anos), foram identificados. Os pacientes receberam TRT por uma média de 36 meses após a PR (1-136 meses) e foram acompanhados por uma média de 13 meses após terem iniciado a suplementação com testosterona (1-99 meses). Os valores médios da testosterona aumentaram de $255 \mathrm{ng} / \mathrm{dL}$ (antes da TRT) para $459 \mathrm{ng} / \mathrm{dL}$ após a TRT $(\mathrm{p}<0,001)$. Não houve aumento nos níveis de PSA após o início da TRT e, dessa forma, não houve diagnóstico de recorrência bioquímica. Os autores concluíram que a TRT foi efetiva no aumento dos níveis séricos de testosterona, sem aumentar os níveis de PSA, em homens hipogonádicos que haviam sido tratados com prostatectomia radical para o CaP.

\section{TRT EM HOMENS SUBMETIDOS À BRAQUITERAPIA PARA 0 TRATAMENTO DO CARCINOMA PROSTÁTICO LOCALIZADO}

O único relato de TRT após braquiterapia foi feito por Sarosdy (33) e compreende uma série de 31 homens com níveis séricos de testosterona variando de 30 a 255 $\mathrm{ng} / \mathrm{dL}$ e sintomas compatíveis com hipogonadismo, que receberam TRT após 0,5 a 4,5 anos da braquiterapia. Este estudo objetivou avaliar o risco de recorrência bioquímica do CaP. Dados obtidos de homens que realizaram braquiterapia transperineal para tratamento do $\mathrm{CaP}$ localizado foram revisados de maneira retrospectiva.

Os detalhes relacionados ao tratamento foram pesquisados, incluindo se a braquiterapia foi usada isoladamente ou em combinação com a radioterapia externa ou bloqueio androgênico. Quando o último foi utilizado, tratou-se de uso de agonista do LHRH (leuprolide ou goserelina) isoladamente ou em combinação com antiandrogênico não esteroidal (bicalutamida) por um período máximo de 12 meses.

A TRT foi iniciada seis ou mais meses após a braquiterapia, após a confirmação de que os níveis séricos de testosterona eram inferiores a $300 \mathrm{ng} / \mathrm{dL}$, além de sintomas, como ondas de calor, fadiga, depressão, disfunção erétil ou diminuição da libido. A TRT foi continuada após em homens que manifestaram resposta clínica benéfica. A escolha do agente farmacológico de eleição dependeu da preferência de cada paciente, apesar de a maioria ter recebido injeções intramusculares (cipionato de testosterona $200 \mathrm{mg}$ ) para evitar o problema da absorção variável dos produtos transdérmicos. O consentimento informado foi distribuído a todos os pacientes. O acompanhamento consistiu de visitas semestrais por cinco anos após a braquiterapia e visitas anuais posteriormente. Em cada visita, foram realizados exame físico e aferição dos níveis séricos de PSA e testosterona total.

Trinta e seis pacientes (de 715 homens que receberam braquiterapia) (5\%) foram incluídos. Cinco pacientes receberam 1 a 3 meses de TRT, a qual foi suspensa após os pacientes relatarem ausência de benefício no controle de sintomas associados. A idade variou de $5 \mathrm{l}$ a 79 anos (mediana $=65$ anos) na época da braquiterapia. A mediana do nível sérico de PSA foi de $5,3 \mathrm{ng} / \mathrm{mL}$, o escore de Gleason mais comum foi o 6 (19 de 31 homens - 61,3\%) e o estágio clínico mais frequente foi o Tlc (20 de 31 pacientes $-64,5 \%$ ). Vinte pacientes realizaram braquiterapia isoladamente, e os restantes 11 pacientes receberam braquiterapia associada à radioterapia externa. O bloqueio androgênico temporário foi utilizado por 8 a 12 meses em 14 pacientes com doença de alto risco. A TRT foi iniciada 0,5 a 4,25 anos (mediana de 2,0 anos) após a braquiterapia. Os níveis de testosterona sérica pré-TRT variaram de 30 a $255 \mathrm{ng} / \mathrm{dL}$ (mediana $=188 \mathrm{ng} / \mathrm{dL})$. A duração da TRT variou de $0,5 \mathrm{a}$ 8,5 anos (mediana $=4,5$ anos). O nível máximo de testosterona durante a TRT variou de 356 a $1.373 \mathrm{ng} / \mathrm{dL}$ (mediana $=498 \mathrm{ng} / \mathrm{dL})$. O acompanhamento pós-braquiterapia variou de 1,5 a 9,0 anos (mediana $=5$ anos). O PSA mais recente foi $<0,1 \mathrm{ng} / \mathrm{mL}$ em 23 pacientes $(74,2 \%),<0,5 \mathrm{ng} / \mathrm{mL}$ em 30 pacientes $(96,7 \%)$ e $<1$ $\mathrm{ng} / \mathrm{mL}$ nos 31 pacientes (100\%). Nenhum paciente interrompeu a TRT em função de recorrência bioquímica ou progressão clínica. Os autores concluíram que a TRT pode ser utilizada, com acompanhamento rigoroso, em homens hipogonádicos que realizaram braquiterapia como forma de tratamento do CaP localizado.

\section{TRT EM HOMENS SUBMETIDOS À RADIOTERAPIA EXTERNA PARA $O$ TRATAMENTO DO CARCINOMA PROSTÁTICO}

A radioterapia externa (EBRT, external beam radiotherapy) é um tratamento potencialmente curativo que pode ser oferecido a homens com o diagnóstico de $\mathrm{CaP}$ localizado. Essa intervenção pode ser precedida por bloqueio androgênico, o que pode resultar em disfunção testicular que pode não melhorar completamente após a suspensão do medicamento antiandrogênico. Reconhece-se que três fatores, incluindo a população idosa com o risco inerente de disfunção do eixo hipotálamo-pitui- 
tário-gonadal, além da supressão iatrogênica da função gonadal (antiandrogênicos) e ao dano testicular pela radiação ionizante, podem ser apontados como etiologia de hipogonadismo após o tratamento do $\mathrm{CaP}$ com radioterapia externa (34).

Recentemente, Morales e cols. (34) publicaram um estudo que descreveu cinco homens com sinais e sintomas de hipogonadismo pós-tratamento de CaP localizado com EBRT, que receberam TRT uma vez que o PSA atingiu o nadir. Esses homens receberam explicações sobre os riscos e potenciais benefícios da TRT e a necessidade de acompanhamento rigoroso. Todos os homens tiveram confirmação histológica do CaP, sem evidências de doença localmente avançada ou metástases a distância. A média do escore de Gleason foi 7 (6-8), a média do PSA pré-EBRT foi $12,8(3,8-28)$ e a média do PSA pré-TRT foi de $0,3 \mathrm{ng} / \mathrm{mL}(<0,1$ a 0,97$)$. Dois pacientes optaram por injeções intramusculares, um por testosterona via oral e dois pela administração transdérmica de testosterona. A duração do acompanhamento durante a TRT foi de 14,6 meses (6-27). Os níveis de testosterona antes da TRT foram de 5,2 nmol/L (1,1$9,2)$; na última visita, os níveis foram de $17,6 \mathrm{nmol} / \mathrm{L}$ $(8,5-32,4)$. Um dos cinco pacientes teve um aumento transitório no PSA, mas nenhum teve nível superior a $1,5 \mathrm{ng} / \mathrm{mL}$. Todos os pacientes relataram melhora significativa nas manifestações clínicas do hipogonadismo. Um paciente morreu de causa não relacionada à neoplasia $\mathrm{e}$ um paciente suspendeu o tratamento em seis meses devido aos efeitos colaterais (cefaleias). Nenhum paciente teve recorrência clínica ou bioquímica do carcinoma prostático e os autores concluíram que este estudo não deveria ser considerado como evidência da segurança da TRT em homens tratados com EBRT para o CaP, em função do pequeno número de pacientes incluídos. Estudos adicionais deveriam ser realizados.

\section{DISCUSSÃO}

O risco de desenvolvimento do carcinoma prostático em homens recebendo TRT não é conhecido (1). A evidência científica atual consiste de estudos clínicos que incluíram poucas centenas de homens, e esses estudos não foram delineados a fim de estabelecer o risco de desenvolvimento de CaP clínico. Nenhum dos ensaios clínicos randomizados placebo-controlados sobre TRT para hipogonadismo exigiram biópsias prostáticas no início ou ao final do estudo. Assim, a prevalência e a incidência de carcinoma prostático oculto não foram es- tudadas. A análise dos estudos prospectivos publicados sobre TRT (meta-análise) revelou apenas cinco casos de carcinoma prostático em 461 homens tratados $(1,1 \%)$, seguidos por um período de seis a 36 meses. Essa taxa de prevalência é semelhante à encontrada na população geral (15). Esses estudos incluíram homens com hipogonadismo com várias causas e graus, que podem ou não ter recebido suplementação de testosterona antes da inclusão nos estudos prospectivos.

Considerando a prevalência de $14 \%$ de carcinoma oculto em homens com níveis séricos baixos de testosterona total ou livre, estimada por Morgentaler e cols. (35), eram esperados 65 casos de câncer em uma série de 461 homens. Dessa forma, o que aconteceu com esses 60 que receberam TRT e não tiveram o diagnóstico de CaP na meta-análise descrita acima? Comparar um estudo retrospectivo da prevalência de carcinoma prostático oculto em homens hipogonádicos com a taxa de prevalência de CaP em meta-análise de ensaios clínicos randomizados pode não ser apropriado. Contudo, o reconhecimento desses resultados conflitantes torna-se, no mínimo, intrigante.

Apesar de serem oriundos de estudos clínicos pequenos, os dados que demonstram a segurança da TRT estão aparecendo, o que tem levado a um aumento na sua indicação. Até o presente momento, um dos tópicos mais controversos é se a TRT seria segura em homens tratados para o carcinoma prostático. Na presente revisão da literatura, foram encontradas cinco pequenas séries, incluindo 110 pacientes (74 pacientes pós-prostatectomia radical/31 pacientes pós-braquiterapia/cinco pacientes pós-radioterapia externa) que receberam TRT após tratamento potencialmente curativo do $\mathrm{CaP}$ $(2,16,32-34)$. Essas pequenas séries de casos não descreveram recorrências bioquímicas durante o período de acompanhamento. Entretanto, elas são inadequadas para embasar de maneira sólida a TRT para essa população selecionada. De outro lado, esse é um tópico atual, haja vista que há um aumento na expectativa de vida da população e na sobrevida dos pacientes tratados para carcinoma prostático. Tal contexto faz com que se tenha um aumento do número de pacientes hipogonádicos que tenham sido tratados com intenção curativa para o CaP no passado.

Recentemente, Shabsigh e cols. (36) publicaram uma revisão sistemática sobre o risco de carcinoma prostático em pacientes hipogonádicos tratados com TRT. Uma pesquisa rigorosa dos bancos de dados MedLine, EMBASE e outras fontes foi conduzida para 
identificar artigos que descrevessem ocorrências de CaP em homens recebendo TRT para o tratamento do hipogonadismo. Os artigos foram avaliados quanto à causalidade entre TRT e CaP, aumento do PSA e achados do exame digital prostático. De 197 artigos relacionados à TRT, 44 encontraram os critérios de inclusão: 11 estudos randomizados, placebo-controlados; 29 estudos não placebo-controlados de homens sem história de CaP; e quatro estudos de homens hipogonádicos com história de CaP. Desses estudos, nenhum demonstrou que a TRT para hipogonadismo aumentou o risco de $\mathrm{CaP}$ ou aumentou o escore de Gleason de CaP detectado em homens tratados versus não tratados. Não houve evidência de que a TRT aumenta o risco de CaP em homens hipogonádicos. Estudos selecionados nesta revisão também demonstraram que a taxa de incidência de CaP não aumentou e não houve progressão de PIN de alto grau para $\mathrm{CaP}$ em homens hipogonádicos tratados com TRT. Além disso, concentrações endógenas de testosterona sérica não estiveram associadas diretamente a mudanças anormais no PSA, e a TRT pareceu ser segura em homens tratados com intenção curativa para o $\mathrm{CaP}(36)$.

\section{CONCLUSÕES}

Pouca atenção foi dada previamente às consequências do hipogonadismo em homens com carcinoma prostático. Estudos demonstrando as consequências deletérias do hipogonadismo, tanto iatrogênico quanto espontâneo, têm sido publicados. $\mathrm{O}$ surgimento de diabetes e síndrome metabólica, além do risco de morte por causas cardiovasculares, está entre as consequências sérias do hipogonadismo. Há evidências cada vez mais claras na literatura médica sobre a segurança e a efetividade da terapia de reposição com testosterona em homens hipogonádicos. Contudo, mais estudos são necessários antes que se possa tecer considerações definitivas em relação ao uso de TRT em homens hipogonádicos que receberam tratamento potencialmente curativo para o carcinoma prostático. Apesar das suas limitações inerentes, as séries de casos publicadas até o momento sugerem que a TRT pode ser considerada em pacientes hipogonádicos, previamente tratados com intenção curativa para o carcinoma prostático de baixo risco, que não tenham evidência de doença ativa.

Declaração: os autores declaram não haver conflitos de interesse científico neste estudo.

\section{REFERÊNCIAS}

1. Rhoden EL, Averbeck MA, Teloken PE. Androgen replacement in men undergoing treatment for prostate carcinoma. J Sex Med. 2008;5(9):2202-8.

2. Agarwal PK, Oefelein MG. Testosterone replacement therapy after primary treatment for prostate carcinoma. J Urol. 2005;173:533.

3. Maggi M, Schulman C, Quinton R, Langham S, Uhl-Hochgraeber $\mathrm{K}$. The burden of testosterone deficiency syndrome in adult men: economic and quality-of-life impact. J Sex Med. 2007;4(4 Pt 1):1056-69.

4. Garos S, Kluck A, Aronoff D. Prostate carcinoma patients and their partners: differences in satisfaction indices and psychological variables. J Sex Med. 2007;4(5):1394-403.

5. Nelson CJ, Choi JM, Mulhall JP, Roth AJ. Determinants of sexual satisfaction in men with prostate carcinoma. J Sex Med. 2007;4(5):1422-7.

6. Huggins C, Hodges CV. Studies on prostatic cancer. I. The effect of castration, of estrogen and androgen injection on serum phosphatases in metastatic carcinoma of the prostate. Cancer Res. 1941;1:293.

7. Stattin P, Lumme S, Tenkanen L. High levels of circulating testosterone are not associated with increased prostate carcinoma risk: a pooled prospective study. Int J Cancer. 2004;108:418.

8. Wiren S, Stocks T, Rinaldi S. Androgens and prostate carcinoma risk: a prospective study. Prostate. 2007;67:1230.

9. Severi G, Morris HA, MacInnis RJ. Circulating steroid hormones and the risk of prostate carcinoma. Cancer Epidemiol Biomarkers Prev. 2006;15(1):86-91.

10. Vatten LJ, Ursin G, Ross RK. Androgens in serum and the risk of prostate carcinoma: a nested case-control study from the Janus serum bank in Norway. Cancer Epidemiol Biomarkers Prev. 1997;6(11):967-9.

11. Chen C, Weiss NS, Stanczyk FZ. Endogenous sex hormones and prostate carcinoma risk: a case-control study nested within the Carotene and Retinol Efficacy Trial. Cancer Epidemiol Biomarkers Prev. 2003;12(12):1410-6.

12. Roddam AW, Allen NE, Appleby P. Endogenous sex hormones and prostate carcinoma: a collaborative analysis of 18 prospective studies. J Natl Cancer Inst. 2008;100(3):170-83.

13. Gann P, Hennekens C, Ma J, Longcope C, Stampfer M. Prospective study of sex hormone levels and risk of prostate carcinoma. $J$ Natl Cancer Inst. 1996;88:1118-26.

14. Parsons JK, Carter HB, Platz EA, Wright EJ, Landis P, Metter EJ. Serum testosterone and the risk of prostate carcinoma: potential implications for testosterone therapy. Cancer Epidemiol Biomarkers Prev. 2005; 14:2257-60.

15. Rhoden EL, Morgentaler A. Risks of testosterone-replacement therapy and recommendations for monitoring. N Engl J Med. 2004;350:482.

16. Kaufman JM, Graydon RJ. Androgen replacement after curative radical prostatectomy for prostate carcinoma in hypogonadal men. J Urol. 2004;172:920.

17. Bhasin S, Cunningham GR, Hayes FJ. Testosterone therapy in adult men with androgen deficiency syndromes: an endocrine society clinical practice guideline. J Clin Endocrinol Metab. 2006;91:1995.

18. van Weerden WM, van Steenbrugge GJ, van Kreuningen A. Assessment of the critical level of androgen for growth response of transplantable human prostatic carcinoma (PC-82) in nude mice. J Urol. 1991;145:631.

19. Pearson OH. Discussion of Dr. Huggins' paper: control of cancers of man by endocrinological methods. Cancer Res. 1957;17:473.

20. Fowler JE Jr, Whitmore WF. The response of metastatic adenocarcinoma of the prostate to exogenous testosterone. J Urol. 1981;126:372. 
21. Prout GR Jr, BrewerWR. Response of men with advanced prostatic carcinoma to exogenous administration of testosterone. Cancer. 1967;20:1871.

22. Morgentaler A. Testosterone and prostate carcinoma: an historical perspective on a modern myth. Eur Urol. 2006;50:935.

23. Marks LS, Mazer NA, Mostaghel E. Effect of testosterone replacement therapy on prostate tissue in men with late-onset hypogonadism: a randomized controlled trial. JAMA. 2006;296:2351.

24. El-Sakka Al, Hassoba HM, Elbakry AM, Hassan HA. Prostatic specific antigen in patients with hypogonadism: effect of testosterone replacement. J Sex Med. 2005;2(2):235-40.

25. Bhasin S, Woodhouse L, Casaburi R. Testosterone dose-response relationships in healthy young men. Am J Physiol Endocrinol Metab. 2001;281:E1172.

26. Cooper CS, Perry PJ, Sparks AE. Effect of exogenous testosterone on prostate volume, serum and semen prostate specific antigen levels in healthy young men. J Urol. 1998;159:441.

27. Loughlin KR, Richie JP. Prostate carcinoma after exogenous testosterone treatment for impotence. J Urol. 1997;157:1845.

28. Curran MJ, Bihrle W. Dramatic rise in prostate-specific antigen after androgen replacement in a hypogonadal man with occult adenocarcinoma of the prostate. Urology. 1999;53:423.

29. Bubley GJ. Is the flare phenomenon clinically significant? Urology. 2001;58:5.
30. Kuhn JM, BillebaudT, Navratil H. Prevention of the transient adverse effects of a gonadotropin-releasing hormone analogue (buserelin) in metastatic prostatic carcinoma by administration of an antiandrogen (nilutamide). N Engl J Med. 1989;321:413.

31. Tomera K, Gleason D, Gittelman M. The gonadotropin-releasing hormone antagonist abarelix depot versus luteinizing hormone releasing hormone agonists leuprolide or goserelin: initial results of endocrinological and biochemical efficacies in patients with prostate carcinoma. J Urol. 2001;165:1585.

32. Khera M, Grober ED, Najari B, Colen JS, Mohamed O, Lamb DJ, et al. Testosterone replacement therapy following radical prostatectomy. J Sex Med. 2009;6(4):1165-70.

33. Sarosdy MF. Testosterone replacement for hypogonadism after treatment of early prostate carcinoma with brachytherapy. Cancer. 2007;109:536.

34. Morales A, Black AM, Emerson LE. Testosterone administration to men with testosterone deficiency syndrome after external beam radiotherapy for localized prostate carcinoma: preliminary observations. BJU Int. 2008. doi:10.1111/j.1464-410X.2008.07882.x

35. Morgentaler A, Bruning CO, DeWolf WC. Occult prostate carcinoma in men with low serum testosterone levels. JAMA. 1996;276:1904.

36. Shabsigh R, Crawford ED, Nehra A, Slawin KM. Testosterone therapy in hypogonadal men and potential prostate cancer risk: a systematic review. Int J Impot Res. 2009;(21):9-23. 